\title{
CYSTS OF UVEAL ORIGIN IN THE VITREOUS*
}

\author{
BY \\ SÜREYYA GÖRDÜREN \\ Department of Ophthalmology, University cf Ankara, Turkey
}

Cysts in the vitreous, originating from the uvea, are very rare; they may be divided into congenital, traumatic, neoplastic, degenerative, and inflammatory. Being usually transparent, they do not impair vision and are often only discovered accidentally in the course of a routine examination.

They are generally unilateral, but Vennin (1910), Litinsky (1931), Perera (1936), and some other authors, have reported bilateral cysts. In the majority of cases, only one cyst can be seen in the same eye. The case to be described is characterized by the presence of two cysts in the same eye, and by signs revealing their possible uveal origin.

\section{Case Report}

A. F., a male aged 20, came to our clinic complaining of poor vision since childhood, and was admitted to the hospital on the discovery of a cyst in his left eye. No previous disease or trauma was reported. Except for a concomitant squint of $10^{\circ}$ involving the right eye, both eyes appeared normal.

Tension in both eyes was $18 \mathrm{~mm}$. $\mathrm{Hg}$ Schiötz.

Retinoscopic examination revealed a myopia of $8.00 \mathrm{D}$. in the right eye, and $5.00 \mathrm{D}$. in the left.

Vision $1 / 50$ in the right eye, and 20/50 in the left, could not be improved.

Slit-lamp examination with dilated pupil disclosed small punctate opacities in the equatorial zone of the lens of each eye.

Right Eye.-The slightiy liquefied vitreous showed, no pigmentation. On ophthalmoscopic examination, a large myopic conus was found extending to the macula, and the slightly depigmented retina presented a tigroid appearance.

Left Eye.-Biomicroscopic examination revealed two regular spherical bodies of $0.5 \mathrm{~mm}$. apparent diameter (one totally transparent and cystic, and the other solid and orange coloured) situated near the posterior pole of the lens (Fig. 1). The two were suspended from two fibrils parallel to each other, and each was connected to a third fibril. They moved with eye movements and returned to their original position when the movement ceased. Two smaller, round, mobile, pigment granules, attached to other thin vitreous fibrils, were located a little above and behind these two bodies.

Although no pigmentation was present in the other eye, numerous smaller and less mobile pigment granules were seen in the inferior quadrant in contact with the posterior surface of the lens. Unlike the fragmentary pigmentation seen in glaucoma, inflammatory, or degenerative conditions, they resembled the flocculi normally observed on the pupillary margin, thus suggesting the possibility that they might similarly have arisen from proliteration of the pigment epithelium. A second cyst, round and translucent, with bright yellowish-orange irregular patches on its surface, was observed at the junction of the anterior $1 / 3$ and the posterior $2 / 3$ of the vitreous. This cyst moved and revolved upon its axis

${ }^{*}$ Received for publication September 28, 1950. 


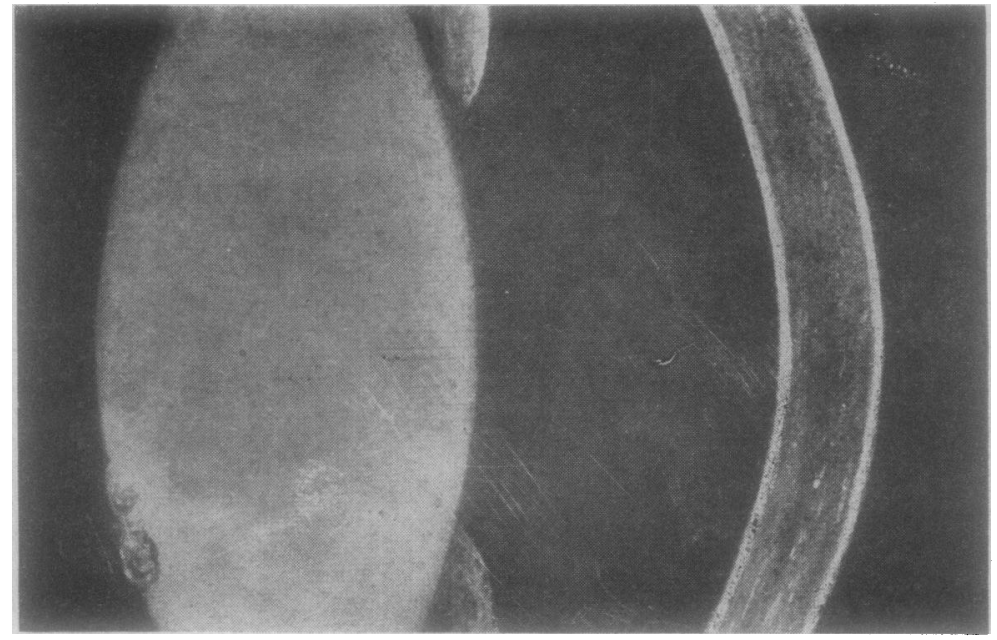

FIG. 1.-Slit-lamp appearance of the cyst and the pigmented body situated near the posterior pole of the lens

with eye movements, and on cessation of these movements, returned to its original position. It had no visible attachment to any part of the eye. No

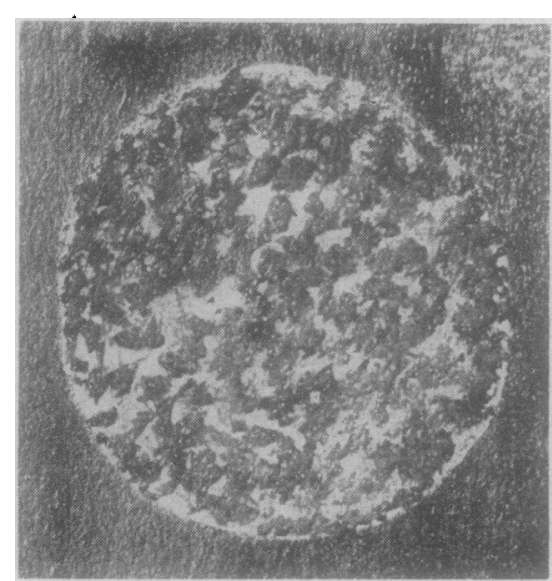

FIG. 2.-Ophthalmoscopic appearance of the large cyst. active movements or the presence of scolex or receptaculum capitis indicating a cysticercus, could be observed, either in the small transparent cyst or in the larger pigmented one.

On ophthalmoscopic examination, the small cyst was not seen, and the large one appeared like a round cage bespattered with small black patches, casting a shadow on the retina. This cyst was brought into sharpest focus with a +12.00 D. lens with direct ophthalmoscopy, and $+2.50 \mathrm{D}$. lens of the auxiliary slide with the binocular ophthalmoscope (Bausch and Lomb), when a yellowish-orange pigmentation and an iridescence varying according to the light conditions was observed (Fig. 2). The disk was oval, being elongated in the vertical direction. A temporal conus and areas of myopic atrophy were noticed in the retina.

No remnants of the hyaloid artery could be seen on the disk or on the posterior surface of the lens. Transillumination revealed no abnormal shadow in the ciliary region. The patient was observed for 6 months and no change could be determined either in the cysts or in the eye itself.

Routine studies of the blood:

$3,600,000$ erythrocytes, 4,600 leucocytes, 3 per cent. eosinophils,

Sedimentation rate $4 \mathrm{~mm}$. in one hour,

Wassermann and Mantoux reactions negative,

Weinberg test positive,

Casoni test negative. 
The ova of Ascaris lumbricoides, Trichocephalus, and Taenia saginata were found in the stools.

Cysts in the vitreous, apart from those of parasitic origin, provoke no irritation, and therefore, require no treatment. Since no pathological examinations have been performed on these cysts, their aetiology and nature has not been established with certainty.

\section{Discussion}

Our case presents bilateral high myopia, more marked in the uninvolved eye. Cysts in the vitreous, supposedly due to myopic retinal degeneration, have been reported by Vennin (1910), but even in his cases a congenital origin cannot be excluded.

In the case here reported, the presence of abundant pigmentation in the anterior part of the vitreous in association with the cysts in the left eye, and their absence in the right or more myopic eye suggest an aetiology other than myopic degeneration. It is felt that these cysts are probably of congenital origin.

The small cyst and the pigment granules were situated in the retrolental space, and the large cyst occupied the site of Cloquet's canal though it contained no remnants of the canal structures. Nevertheless this position corresponds to the primary vitreous and suggests a developmental anomaly. It is possible that a proliferation of the ciliary epithelium may undergo cystic degeneration and detach itself into the vitreous, during intrauterine or post-natal life. Berliner (1949) claimed that pigmented cysts are not necessarily of uveal origin. But in our case the adjacency of the small cyst and the pigmented spherical body, their similarity, and the presence of numerous pigment granules nearby, suggest that each is probably of uveal origin.

\section{REFERENCES}

Berliner, M. L. (1949). "Biomicroscopy of the Eye", vol. 2. Hoeber, New York.

Litinsky, G. A. (1931). Klin. Mbl. Augenheilk., 87, 205.

Perera, C. A. (1936). Arch. Ophthal., Chicaso, 16, 1015.

VenNin (1910). Lyon méd., 114, 974. 